\title{
Long-Term Results of External Upper Esophageal Sphincter Myotomy for Oropharyngeal Dysphagia
}

\author{
Martijn P. Kos · Eric F. David • \\ Elly C. Klinkenberg-Knol · Hans F. Mahieu
}

Published online: 17 September 2009

(c) The Author(s) 2009. This article is published with open access at Springerlink.com

\begin{abstract}
The aim of this work was to assess the efficacy of external myotomy of the upper esophageal sphincter (UES) for oropharyngeal dysphagia. In the period 19912006, 28 patients with longstanding dysphagia and/or aspiration problems of different etiologies underwent UES myotomy as a single surgical treatment. The main symptoms were difficulties in swallowing of a solid-food bolus, aspiration, and recurrent incidents of solid-food blockages. Pre- and postoperative manometry and videofluoroscopy were used to assess deglutition and aspiration. Outcome was defined as success in the case of complete relief or marked improvement of dysphagia and aspiration and as failure in the case of partial improvement or no improvement. Initial results showed success in 21 and failure in 7 patients. The best outcomes were observed in patients with dysphagia of unknown origin, noncancer-related iatrogenic
\end{abstract}

M. P. Kos · H. F. Mahieu

Department of Otolaryngology/Head and Neck Surgery, VU University Medical Center, Amsterdam, The Netherlands

M. P. Kos (ه)

Department of Otolaryngology, Waterland Hospital, P.O. Box 250, 1440 AG Purmerend, The Netherlands e-mail: martijn.kos@gmail.com

E. F. David

Department of Radiology, VU University Medical Center, Amsterdam, The Netherlands

E. C. Klinkenberg-Knol

Department of Gastroenterology, VU University Medical Center, Amsterdam, The Netherlands

Present Address:

H. F. Mahieu

Department of Otolaryngology/Head and Neck Surgery,

Meander Medical Center, Amersfoort, The Netherlands etiology, and neuromuscular disease. No correlation was found between preoperative constrictor pharyngeal muscle activity and success rate. After follow-up of more than 1 year, 20 patients were marked as success and 3 as failure. All successful patients had full oral intake with a normal bolus consistency without clinically significant aspiration. We conclude that in select cases of oropharyngeal dysphagia success may be achieved by UES myotomy with restoration of oral intake of normal bolus consistency.

Keywords Oropharyngeal dysphagia - UES myotomy · Muscular dysphagia $\cdot$ Neurogenic dysphagia .

Videofluoroscopy · Manometry · Deglutition · Deglutition disorders

\section{Introduction}

The upper esophageal sphincter (UES) remains tonically contracted between swallows and acts as an additional barrier preventing influx of air during inspiration and protection of the upper airway from reflux from the esophagus and stomach. Normal physiology of deglutition requires the opening of the esophageal inlet to enable passage of the bolus from the pharynx into the esophagus. This opening of the esophageal inlet is achieved by a combination of (1) elevation and anterior displacement of the larynx, which assists in the esophageal inlet being pulled open, (2) relaxation of the UES, and (3) passive dilatation of the esophageal inlet as a consequence of the propulsion of the bolus being pushed downward by the peristaltic contraction of the pharyngeal constrictor muscles [1]. Failure of UES relaxation or other forms of cricopharyngeal dysfunction and diminished pharyngeal constrictor activity lead to obstruction of bolus passage [2] 
and can result in aspiration of food and saliva. Usually, deglutition of solid bolus is more affected than deglutition of liquids because a large opening of the esophageal inlet is required to enable the passage of a solid bolus, whereas a minor opening of the esophageal inlet will allow passage of liquids. Various conditions affect the complex coordinated actions of neuromuscular structures in the hypopharyngeal, laryngeal, and UES regions. They can be divided in neurogenic, myogenic, idiopathic, and iatrogenic causes [3, 4]. Adaptation of food bolus consistency can be the first step in treating oropharyngeal dysphagia. In severe cases replacement of oral alimentation by nutrition via a gastrostomy can be considered. However, this is not always a satisfactory alternative because swallowing of saliva persists and the patient is denied the quality-of-life and social aspects associated with the enjoyment of eating.

UES myotomy is the most frequently used surgical technique to treat oropharyngeal dysphagia and aspiration. The goal of the procedure is diminished pharyngeal constrictor function as well as reduced resistance of the functionally obstructing UES which will facilitate transfer of the bolus from the pharynx into the cervical esophagus. The functional UES unit is not identical to the anatomical UES and is formed by the cricopharyngeal muscle, the last few centimeters of the inferior constrictor muscles, and the first few centimeters of the cervical esophagus. The surgical intervention of UES myotomy consists of sectioning all the muscles that constitute the functional UES unit. Although myotomy is directed at the functional UES unit, oropharyngeal dysphagia is commonly associated with impairment of the pharyngeal musculature as the major pathophysiological factor and is less frequently caused by true cricopharyngeal dysfunction. Because correction of the weak or absent pharyngeal musculature is not possible, reduction of the resistance of the UES by means of a myotomy is the most logical approach to facilitate bolus propulsion. This will overcome the reduced resistance of the UES to successfully open the esophageal inlet. Many consider at least some remaining hypopharyngeal muscular activity a key factor in successful outcome of any intervention for oropharyngeal dysphagia. Absence of pharyngeal constrictor activity and rapid muscular deterioration is considered a contraindication for UES myotomy by some authors [3, 4].

The first UES myotomy was described by Kaplan in 1951 [5]. The surgery was carried out using an open transcervical approach in a patient following poliomyelitis. This was and continues to be the technique of choice for many head and neck surgeons, although since 1994 endoscopic laser-assisted transmucosal myotomy has been used increasingly.

We have carried out a retrospective study on 28 consecutive oropharyngeal dysphagic patients who underwent external UES myotomy as the only surgical intervention to treat their dysphagia.

\section{Patients and Methods}

\section{Patients}

Following approval of the human studies review committee, a retrospective analysis was performed of all UES myotomies by the senior author (HFM) from 1991 to 2006 . More than 200 patients had undergone UES myotomy. Endoscopic transmucosal myotomy in combination with endoscopic Zenker's diverticulum treatment was performed in 111 cases, endoscopic transmucosal myotomy as monotherapy in 9, external myotomy following laryngectomy in 40 , external myotomy in combination with other surgical corrections (e.g., laryngeal suspension) in 20, and external myotomy as a single surgical therapy for oropharyngeal dysphagia in 28 . The last 28 patients constituted this study's population and consisted of 15 men and 13 women with a mean age of 60.3 years (range $=33-89$ ). Five patients had progressive myogenic disease ( 2 oculopharyngeal muscular dystrophy, 2 inclusion body myositis, 1 hereditary myopathy), 4 patients had suffered central nervous system (CNS) damage (3 stroke, 1 radiation induced), 4 patients had undergone extensive treatment for head and neck cancer with postoperative radiotherapy (2 supraglottic laryngectomy, 1 maxillectomy, 1 total glossectomy), 4 patients had noncancer-related iatrogenic causes for their dysphagia ( 3 had cranial nerve damage after neck surgery for glomus tumor or neurofibromatosis, 1 fibrosis following orthopedic surgery anterior to the cervical vertebrae), and in 11 patients the cause of dysphagia remained unknown. This last patient group consisted of patients in whom no anatomical or apparent neurological disorder could be found but who nevertheless experienced functional deficits of pharyngeal swallowing. On fluoroscopy and manometry these patients had impaired propulsion of the bolus due to either a weakness of the constrictor pharyngeus musculature or incomplete relaxation of the UES or both.

\section{Methods}

Preoperative and Postoperative Workup

The following examinations were routinely performed: preoperatively: fluoroscopy, functional endoscopic evaluation of swallowing (FEES)[6], and manometry; postoperatively: fluoroscopy, manometry, and FEES only on indication. Laryngeal function was assessed by means of 
videolaryngostroboscopic examinations both preoperatively and postoperatively.

Esophageal manometry was performed using the UPS2020 stationary measurement system (MMS, Enschede, the Netherlands). The pressure was measured with the Unisensor Microtip catheter type 8304-00-9980-D with three pressure transducers $5 \mathrm{~cm}$ from each other. The UES pressure and relaxation were calculated as well as the maximal amplitude of the hypopharyngeal contractions.

Twenty-four-hour double-probe esophageal pH-metry was performed to exclude preexisting reflux if suspected. Preexisting severe reflux was considered a contraindication because of the increased risk of aspiration of gastric contents following reduction of the UES resistance which is considered one of the barriers against laryngopharyngeal reflux.
Data on laryngeal elevation and aspiration were collected by fluoroscopy and data on hypopharyngeal constrictor activity and UES relaxation by manometry. Contrary to some other authors, we did not consider the manometrically assessed absence of hypopharyngeal constrictor activity a contraindication for UES myotomy.

Outcome Measure

Outcome was defined a success if complete relief or marked improvement of symptoms occurred and failure if there was partial improvement or no improvement. Complete relief was recorded when the patient no longer noticed any swallowing disorder and was able to totally fulfill his nutritional needs by oral intake with a diet of normal consistency and no aspiration. Marked improvement was

Table 1 Preoperative characteristics and outcome for different etiologies

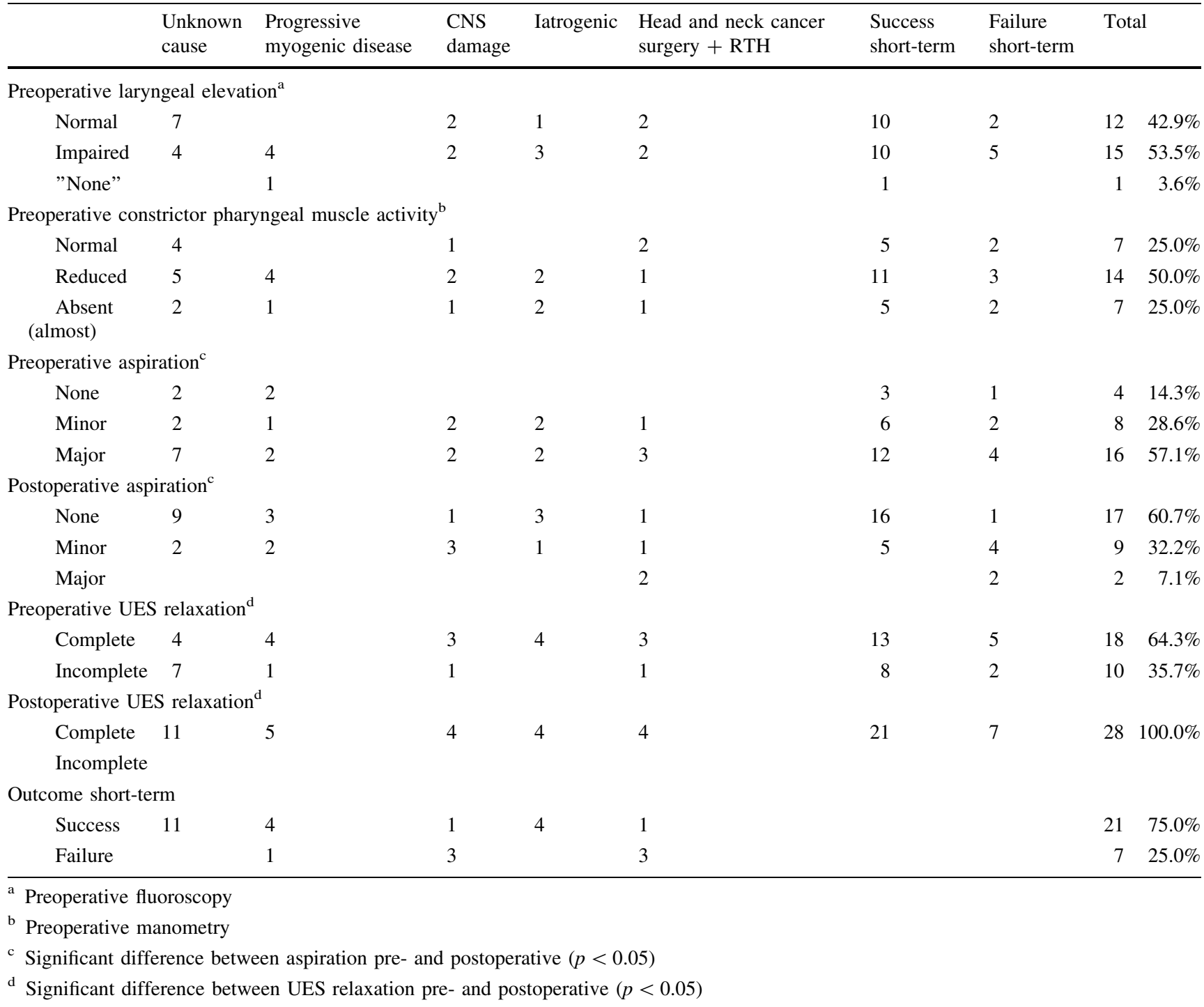


defined as when the patient reported a marked improvement in deglutition but showed some persistence of functional disorders that the patient considered to be minor (e.g., incidental minor aspiration or small parts of a solid bolus such as rice or crumbs that required the drinking of water or other liquids to flush the small particles into the esophagus) and was able to totally fulfill his nutritional needs by oral intake without clinical significant aspiration. Partial improvement was defined as when the patient reported that it was easier to swallow one type of food consistency but continued to have difficulties with other food consistencies, or that oral intake had improved but still was not sufficient for nutritional needs. No improvement was defined as when the patient noticed no changes in swallowing difficulties, had restricted or no oral intake, or had persistent significant aspiration.

If an additional procedure was required to alleviate the dysphagia complaints, the initial myotomy was also scored as a failure.

\section{Statistical Analysis}

Correlation of preoperative variables and outcome was studied with a Spearman test and the difference between pre- and postoperative variables was studied with a Wilcoxon test, both with a $95 \%$ confidence interval. Only significant differences are noted in Table 1.

\section{Operative Procedure}

The surgical procedure starts with an endoscopy to assess the larynx, the pharynx, and the esophagus. A tube with an inflatable balloon or cuff (e.g., Sengstaken tube No. 16, Rusch AG, Kernen, Germany) is then introduced into the esophageal entrance to facilitate the UES myotomy. A leftsided approach to the UES is preferred because the esophagus is usually located slightly left of the trachea and the midline and this enables a better exposure of the UES. The most important complication of external UES myotomy is a recurrent nerve palsy. The risk of developing a recurrent nerve palsy later as a consequence of other pathology is much higher on the left side than on the right. If paralysis of the recurrent laryngeal nerve is already present, then the myotomy should consequently be performed on that side.

A J-shaped incision is made along the anterior border of the sternocleidomastoid muscle, curving toward the midline 1-2 $\mathrm{cm}$ above the sternum. The omohyoid muscle and, if necessary, the superior thyroid artery are transsected for a good exposure. The head is tilted to the contralateral side and the UES myotomy is performed, extending from the lower thyropharyngeal musculature, through the cricopharyngeal muscle, and down to the longitudinal fibers of the

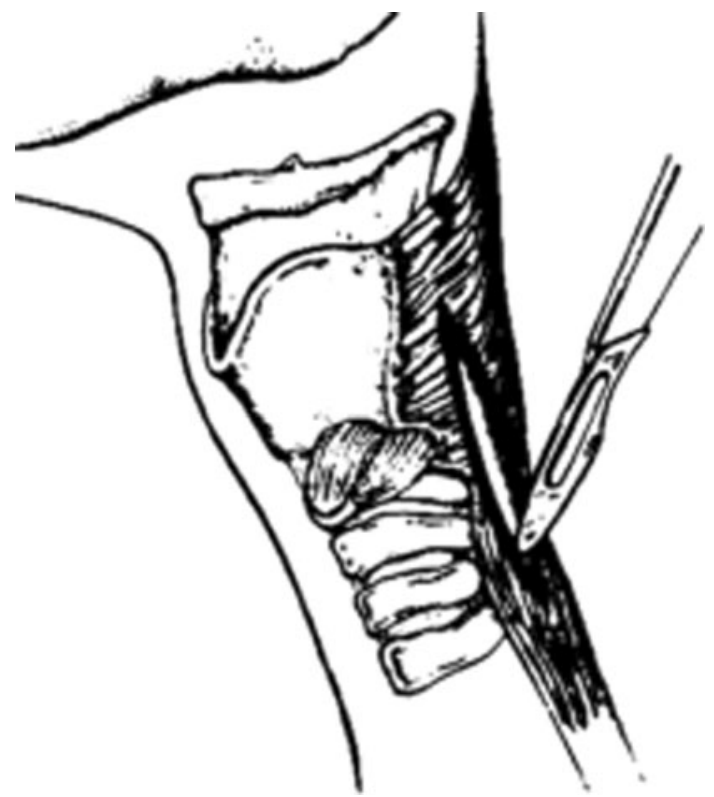

Fig. 1 UES myotomy is performed extending from the lower constrictor pharyngeal musculature, through the cricopharyngeal muscle, down to the longitudinal fibers of the upper esophageal musculature

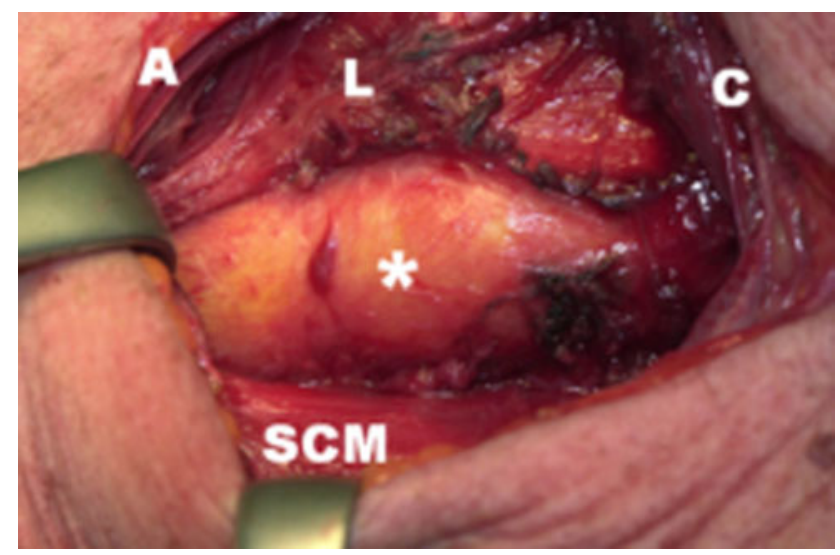

Fig. 2 Image after the sectioning of the UES muscles with an inflated balloon (asterisk) endoluminally positioned in the esophageal entrance. $\mathrm{A}=$ anterior; $\mathrm{SCM}=$ sternocleidomastoid muscle, $\mathrm{C}=$ cranial, $\mathrm{L}=$ larynx

upper esophageal musculature (usually resulting in a total myotomy length of 5-6 cm, Fig. 1). This procedure is facilitated by the inflated balloon in the UES which stretches the muscle fibers and thus allows for very precise sectioning of the UES musculature (Fig. 2). After myotomy the balloon is deflated (Fig. 3). While the balloon is being retracted from the mouth, air is blown through the tube, with saline placed in the external wound, this enables an additional check of the integrity of the UES mucosa. A minor perforation can be found by the escape of air bubbles. Before closure of the neck, a nasogastric feeding tube 


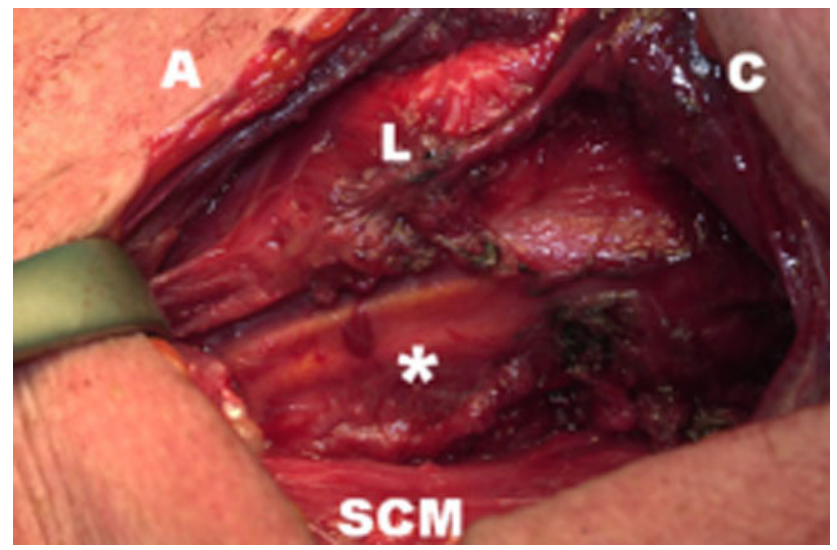

Fig. 3 Image after the sectioning of the UES muscles with the balloon (asterisk), endoluminally positioned in the esophageal entrance, deflated

is placed which is carefully guided through the pharynx and UES by external palpation and gentle pressure of the surgeon's finger in the opened neck. All patients had a strict nonoral intake policy the first two postoperative days.

Swallowing rehabilitation starts on the third postoperative day if no signs of perforation or local infection have occurred. In the first days of rehabilitation edema can interfere with the swallowing act, but prolonged delay of swallow training is not a good idea because of the possible development of local fibrosis and consequently stenosis of the UES. Patients left the hospital after a safe and adequate oral intake had been achieved. If they failed to achieve a sufficiently safe oral intake despite extensive postoperative swallowing rehabilitation, PEG feeding or adequate dietary adjustments might be necessary so that the patient could safely manage at home.

Surgical and clinical notes were carefully studied for postoperative complications. Postoperative fever was defined as one measurement of $>38.5^{\circ} \mathrm{C}$ or 3 days at $>38.0^{\circ} \mathrm{C}$.

\section{Results}

All 28 patients suffered from severe dysphagia that consisted of severe problems with passage of a solid bolus with or without recurrent incidents of solid-food impactions resulting in episodes of aspiration. In 17 patients extensive preoperative weight change had been documented. In the year prior to surgery five patients had no weight loss, seven patients had lost up to $5 \mathrm{~kg}$, three patients up to $10 \mathrm{~kg}$, and two patients up to $15 \mathrm{~kg}$. Three patients also had velopharyngeal insufficiency with nasopharyngeal reflux as a sign of their neuromuscular disease. Six patients were gastrostomy-dependent preoperatively. Four other patients had chosen to remain on oral nutrition despite episodes of aspiration pneumonia. In five patients laryngeal immobility was seen preoperatively, three cases on the left side and two on the right side. The latter two cases were the only patients in whom the UES myotomy was performed on the right side. One patient previously underwent dilatation of UES and esophagus which had provided temporary relieve of his dysphagia. One patient previously had undergone two botulinum toxin injections into the UES as a chemical UES myotomy, which initially had been considered the treatment of choice because of his advanced age ( 88 years) but it only partly alleviated his dysphagic problems. He requested an external UES myotomy which markedly improved his dysphagia and aspiration.

Preoperative functional examinations (Table 1) in the 28 patients demonstrated decreased pharyngeal propulsion in 18 cases, UES dysfunction in 6 patients, and dysfunction of both in 4 patients. Twelve patients had normal, 15 impaired, and 1 no laryngeal elevation preoperatively. Seven patients had normal, 14 reduced, and 7 absent/ almost absent hypopharyngeal constrictor activity preoperatively. In 18 patients UES relaxation was complete and in 10 it was incomplete preoperatively. Preoperatively, 16 patients $(57 \%)$ were judged by fluoroscopy to suffer major aspiration; postoperatively, only 2 patients (7\%) still suffered major aspiration and remained PEG-dependent. Six patients suffered from repeated episodes of aspiration pneumonia preoperatively. In all patients postoperative manometry demonstrated complete loss of UES pressure and thus a technically successfully performed UES myotomy procedure.

An overview of the results is presented in Table 1. The mean follow-up was 3.7 years (median $=2.6$ years, range $=0.2-16.9)$. Initial results after $1-3$ months showed a complete success in 2, marked improvement in 19, partial improvement in 3, and no improvement in 4 patients. Initial outcome was considered a success in $21(75 \%)$ and a failure in 7 (25\%) patients. The long-term outcome after a follow-up period of more than 1 year remained successful for all 19 patients who initially were successful and could be followed (the other 2 patients died within the first year). One patient who was initially unsuccessful 1 month following UES myotomy eventually became successful after a prolonged period of swallowing rehabilitation. In all successful cases full oral intake of a normal bolus consistency and no significant aspiration was achieved. Two of the patients with failed myotomies could not sustain any oral intake and remained on PEG feeding, and the other patients with failed myotomies achieved oral intake with a diet of modified consistency.

Five patients were lost to follow-up within the first year: One patient was lost to follow-up (short-term outcome: no improvement), one died of locoregional recurrence of head and neck cancer (short-term outcome: partial 
improvement), and three died of lung malignancy (shortterm outcome: 1 complete relief, 2 no improvement).

The short-term success rate in patients with progressive myogenic disease $(n=5)$ was $80 \%$, in dysphagia of unknown cause $(n=11) 100 \%$, in patients with CNS damage $(n=4), 25 \%$; in patients who underwent extensive head and neck cancer surgery with radiotherapy $(n=4), 25 \%$; and in the noncancer-related iatrogenic group ( $n=4), 100 \%$.

Of all patients who were followed up for more than 1 year $(n=23)$, only one patient changed from a shortterm failure into a long-term success, and none of the shortterm successes $(n=21)$ changed into a long-term failure. It therefore seems safe to assume that those patients who were lost to follow-up would have had the same long-term outcome as their short-term outcome. This would therefore result in an overall long-term outcome of $79 \%$ success (22 of 28 patients).

\section{Hospital Admission and Complications}

The median postoperative admittance was 6 days (range $=3-56$ days), which included an extensive swallowing rehabilitation program. Two patients were hospitalized for more than 15 days, not because of sequellae of the UES myotomy but because of additional surgical procedures related to their initial pathology. Eight patients developed postoperative fever but no signs of local wound infection, fistulization, or abscess were observed. One of them suffered from aspiration pneumonia in the postoperative period and fully recovered following antibiotic treatment. During surgery a small perforation of the mucosa was detected and consequently sutured in two patients. These two patients were observed for a slightly longer postoperative period and received prophylactic antibiotics for a week. On postoperative videofluoroscopy, both patients had good bolus passage and no signs of fistulization. No other complications were seen. More specifically, no new recurrent nerve palsies were found postoperatively.

\section{Analysis of Failures}

Two patients with failed myotomies had previously undergone a supraglottic laryngectomy with postoperative radiotherapy. In one of them additional laryngeal suspension was performed following the failed UES myotomy, which also proved unsuccessful. This patient preferred PEG dependency rather than total laryngectomy. In the other patient aspiration persisted until he died of lung metastasis 10 months after UES myotomy. One other patient with failed myotomy had undergone a maxillectomy with postoperative radiotherapy. He remained with severe aspiration, could not sustain full oral intake, and died after just more than 1 year of locoregional recurrence. One OPMD patient with initially a failed myotomy noticed improvement in bolus passage after a prolonged period of swallowing rehabilitation and had a successful outcome in the long term. Two other patients with failed myotomies had initially suffered a cerebrovascular accident. They continued to have poor passage of a solid bolus and could sustain only liquid intake. Additional dilatation of the UES because of minor stenosis in one of them could not improve the poor bolus passage. This patient refused additional laryngeal suspension. The other stroke patient was diagnosed with a pulmonary malignancy and died soon afterward while contemplating additional laryngeal suspension. The last patient with a failed myotomy had CNS damage following radiotherapy for a meningioma. He still had poor bolus passage 1 month following the myotomy and was lost to follow-up.

\section{Discussion}

As our results demonstrate, UES myotomy is a safe technique and successful in a majority of cases with oropharyngeal dysphagia and/or aspiration. Our long-term success rate of more than $75 \%$ is in line with other studies $[3,7]$. Often it is said [8] that absence of hypopharyngeal constrictor activity is a contraindication for UES myotomy. In contrast to this statement, our results demonstrate that even in cases with absent or almost absent hypopharyngeal constrictor activity, UES myotomy can be successful. Our results show a success rate of $71 \%$ in diminished or absent constrictor activity, $79 \%$ in reduced constrictor activity, and $71 \%$ in normal constrictor activity. We can therefore state that absent hypopharyngeal constrictor activity was no predictor for outcome in our study. In the publications where absent pharyngeal constrictor activity is considered a contraindication for UES myotomy [3, 4], no alternative treatment is proposed for these cases; thus, it remains unclear what had been done for the patients who lacked constrictor activity in those studies.

The most important prognostic factor for success in our study was the etiology of dysphagia. Neither preoperative manometry (UES relaxation and hypopharyngeal constrictor activity) nor preoperative videofluoroscopy (laryngeal elevation and aspiration) could differentiate between successful outcome and failure.

Our study demonstrates that UES myotomy is indicated for patients with no apparent cause of dysphagia (100\% success) or for a group of patients with noncancer-related iatrogenic etiology of oropharyngeal dysphagia (100\% success). Following CNS damage or extensive head and neck cancer surgery with postoperative radiotherapy, 
patients show poor results in our study (success rate of $25 \%$ ) and these cases therefore do not seem to be favorable for UES myotomy.

The explanation of why the results of UES myotomy are good in patients with muscular disease and in patients without evident disease but with obviously impaired propulsion in the pharyngeal phase as observed on fluoroscopy and manometry is that a UES myotomy facilitates propulsion by reducing the force required to push the bolus through the UES. If other factors such as neurological deficits resulting in uncoordination of the swallowing act or anatomical obstructions resulting from scar development play an important role in the dysphagia, the mere reduction of resistance in the UES by a myotomy is likely to be less effective.

Patient selection is essential and requires a complete medical history and clinical examination, including functional endoscopic examination of swallowing (FEES) [9], videofluoroscopy, and manometry. Videofluoroscopy can demonstrate dysfunction of the UES, demonstrate laryngeal elevation, indicate weakness of the base of the tongue, propelling of the bolus by the constrictor muscles, and indicate if penetration or aspiration occurs from lack of laryngeal protection or stasis. However, it has a very high inter- and intraobserver variability on all aspects but aspiration $[10,11]$. Additional manometry is essential to quantify the results of videofluoroscopy. Ideally, these two procedures would be performed in combination as manofluoroscopy in order to visualize the right position of the pressure probes [12]. This technique was not available at our institution. Simultaneous EMG recordings of the hypopharyngeal constrictor and cricopharyngeal muscle are used by some, and though more invasive, they demonstrate uncoordination in the pharyngoesophageal segment [13]. Esophagogastroscopy and 24-h pH-metry are usually performed only on indication [14]. We consider only severe gastroesophageal reflux to be a contraindication for UES myotomy.

We feel that it is important to perform videofluoroscopy and manometry postoperatively to evaluate the dysphagia status and determine whether the myotomy has been complete. If the result of UES myotomy is not successful because of insufficient pharyngeal propelling combined with insufficient laryngeal elevation, additional laryngeal suspension can be considered [15].

A causative therapeutic approach to oropharyngeal dysphagia is seldom possible (e.g., steroids in polymyositis [16], noninclusion body type), so the first symptomatic approach can be an adaptation in food consistency[3]. Functional treatment is indicated if dysphagia is longstanding and moderate to severe. Especially in cases with muscular disease, it has been proven to be the most important aspect in survival, which is primarily linked to aspiration and pulmonary function [17].

In oculopharyngeal muscular dystrophy (OPMD), UES myotomy has shown excellent results. Coiffier [7] describes a success rate of $90 \%$ in 39 OPMD patients. Our results in five patients with progressive myogenic disease also showed an excellent success rate short and long term. It is important to realize that in cases of slowly progressive neuromuscular disease, alleviation of dysphagia by UES myotomy can be no more than temporary but can give many years of relief from dysphagic problems. However, in rapidly progressive neuromuscular disease, for example amyotrophic lateral sclerosis, UES myotomy is not indicated.

Chemical UES myotomy by use of botulinum toxin can be helpful as a diagnostic treatment and can be indicated in patients with high comorbidity. It is, however, often given under general anesthesia and repetitive treatments are needed[18]. Dilatation usually gives temporary relief and is indicated only in cases where fibrosis of the UES unit is expected[19]. Endoscopic myotomy, an adaptation of the endoscopic laser Zenker's diverticulotomy, has increased in popularity in the last decade[20]. It requires less surgical time and a shorter postoperative hospital stay than external myotomy. Lim[6] describes an overall success rate of $86 \%$ in 44 endoscopically treated patients. Three of 11 patients with aspiration continued to have aspiration after myotomy. Halvorson and Kuhn[21] describe a success rate of $78 \%$ with the endoscopic technique. Institutions that perform endoscopic Zenker's diverticulotomy can easily adjust their technique and perform endoscopic UES myotomy. With extensive experience in both techniques, we prefer the external UES myotomy as the treatment of choice for oropharyngeal dysphagia. It is our considered opinion that the accuracy of sectioning the muscles over the entire length of the functional UES unit is better and the risk of local stenosis is less with the external approach. Furthermore, if biopsy of the UES is required to confirm a neuromuscular diagnosis or if a concomitant procedure such as a laryngeal suspension or thyroplasty is performed, external UES myotomy is indicated. Like some other authors, we use endoscopic UES myotomy in cases of recurrent dysphagia after a previous external UES myotomy[7] and in cases with poor quality of the skin of the neck or poor exposure of the neck. Known potential risks of external myotomy are wound infection, pharyngocutaneous fistula, and paralysis of the recurrent laryngeal nerve[22]. In our study we observed none of these complications. Dauer[15] reports one pharyngocutaneous fistula in eight patients treated with an external myotomy. Larger studies have not reported this complication[3, 7]. 


\section{Conclusion}

In selected cases of oropharyngeal dysphagia, external UES myotomy proves to be a safe and effective procedure. There is no clear correlation between a successful outcome and remaining function of the pharyngeal constrictor musculature. Absence of pharyngeal constrictor activity is therefore no contraindication for the procedure. The procedure is very effective in cases of muscular disease, idiopathic dysphagia, and iatrogenic cranial nerve damage following neck surgery. The outcome after UES myotomy is poor for patients who have had a stroke or other CNS damage and extensive head and neck cancer surgery with postoperative radiotherapy.

Acknowledgment The authors thank R. J. Toohill (Medical College of Wisconsin, Milwaukee) for reviewing the manuscript.

Open Access This article is distributed under the terms of the Creative Commons Attribution Noncommercial License which permits any noncommercial use, distribution, and reproduction in any medium, provided the original author(s) and source are credited.

\section{References}

1. Kahrilas PJ, Dodds WJ, Dent J, Logemann JA, Shaker R. Upper esophageal sphincter function during deglutition. Gastroenterology. 1988;95:52-62.

2. Cook IJ. Cricopharyngeal function and dysfunction. Dysphagia. 1993:8:244-51. doi:10.1007/BF01354546.

3. St. Guily JL, Perie S, Willig TN, Chaussade S, Eymard B, Angelard B. Swallowing disorders in muscular diseases: functional assessment and indications of cricopharyngeal myotomy. Ear Nose Throat J. 1994;73:34-40.

4. Kelly JH. Management of upper esophageal sphincter disorders: indications and complications of myotomy. Am J Med. 2000; 108:43S-6S. doi:10.1016/S0002-9343(99)00334-4.

5. Kaplan S. Paralysis of deglutition, a post-poliomyelitis complication treated by section of the cricopharyngeus muscle. Ann Surg. 1951;133:572-3. doi:10.1097/00000658-195113340-00021.

6. Lim RY. Endoscopic $\mathrm{CO}_{2}$ laser cricopharyngeal myotomy. J Clin Laser Med Surg. 1995;13:241-7.

7. Coiffier L, Périé S, Laforêt P, Eymard B, St. Guily JL. Long-term results of cricopharyngeal myotomy in oculopharyngeal muscular dystrophy. Otolaryngol Head Neck Surg. 2006;135:218-22. doi: 10.1016/j.otohns.2006.03.015.

8. Cook IJ, Kahrilas PJ. AGA technical review on management of oropharyngeal dysphagia. Gastroenterology. 1999;116:455-78. doi:10.1016/S0016-5085(99)70144-7.
9. Leder SB, Acton LM, Lisitano HL, Murray JT. Fiberoptic endoscopic evaluation of swallowing (FEES) with and without blue-dyed food. Dysphagia. 2005;20:157-62. doi:10.1007/s00455005-0009-x.

10. Scott A, Perry A, Bench J. A study of interrater reliability when using videofluoroscopy as an assessment of swallowing. Dysphagia. 1988;13:223-7. doi:10.1007/PL00009576.

11. Stoeckli SJ, Huisman TA, Seifert B, Martin-Harris BJ. Interrater reliability of videofluoroscopic swallow evaluation. Dysphagia. 2003;18:53-7. doi:10.1007/s00455-002-0085-0.

12. Olsson R, Kjellin O, Ekberg O. Videomanometric aspects of pharyngeal constrictor activity. Dysphagia. 1996;11:83-6. doi: 10.1007/BF00417894.

13. Elidan J, Shochina M, Gonen B, Gay I. Electromyography of the inferior constrictor and cricopharyngeal muscles during swallowing. Ann Otol Rhinol Laryngol. 1990;99:466-9.

14. Ford CN. Evaluation and management of laryngopharyngeal reflux. JAMA. 2005;294:1534-40. doi:10.1001/jama.294.12.1534.

15. Kos MP, David EF, Aalders IJ, Smit CF, Mahieu HF. Long-term results of laryngeal suspension and upper esophageal sphincter myotomy as treatment of life-threatening aspiration. Ann Otol Rhinol Laryngol. 2008;117:574-80.

16. Kagen LJ, Hochman RB, Strong EW. Cricopharyngeal obstruction in inflammatory myopathy (polymyositis/dermatomyositis). Arthritis Rheum. 1985;28:630-6. doi:10.1002/art.1780280606.

17. Hill M, Hughes T, Millford C. Treatment for swallowing difficulties (dysphagia) in chronic muscle disease. Cochrane Database Syst Rev. 2004;CD004303.

18. Moerman MB. Cricopharyngeal Botox injection: indications and technique. Curr Opin Otolaryngol Head Neck Surg. 2006;14: 431-6. doi:10.1097/MOO.0b013e328010b85b.

19. Hatlebakk JG, Castell JA, Spiegel J, Paoletti V, Katz PO, Castell DO. Dilatation therapy for dysphagia in patients with upper esophageal sphincter dysfunction-manometric and symptomatic response. Dis Esophagus. 1998;11:254-9.

20. Takes RP, van den Hoogen FJ, Marres HA. Endoscopic myotomy of the cricopharyngeal muscle with $\mathrm{CO}_{2}$ laser surgery. Head Neck. 2005;27:703-9. doi:10.1002/hed.20201.

21. Halvorson DJ, Kuhn FA. Transmucosal cricopharyngeal myotomy with the potassium-titanyl-phosphate laser in the treatment of cricopharyngeal dysmotility. Ann Otol Rhinol Laryngol. 1994;103:173-7.

22. Brigand C, Ferraro P, Martin J, Duranceau A. Risk factors in patients undergoing cricopharyngeal myotomy. Br J Surg. 2007; 94:978-83. doi:10.1002/bjs.5760.

M. P. Kos MD

E. F. David MD

E. C. Klinkenberg-Knol MD, $\mathrm{PhD}$

H. F. Mahieu MD, PhD 